\title{
Facomatosis pigmentovascular tipo cesioflamea: a propósito de un caso
}

\author{
María del Pilar Pereira-Gómez'; Manuel Martínez-Gómez²; María Natalia Mejía- \\ Barrenche $^{3}$
}

\section{RESUMEN}

La facomatosis pigmentovascular tipo cesioflamea se caracteriza por la presencia de una malformación vascular capilar superficial, que coexiste en la misma superficie cutánea con una melanocitosis dérmica o un nevo anémico. A su vez, esta entidad puede estar asociada a alteraciones de índole sistémico, especialmente del sistema nervioso central. Su incidencia y prevalencia son desconocidas por su baja frecuencia; sin embargo, reviste de gran importancia para los profesionales de la salud relacionados con la atención de recién nacidos. A continuación, se describe el caso de un neonato a término de 5 días de vida, que nació con una mácula azul-grisácea de gran extensión, superpuesta a una mácula eritematoviolácea, que afectaba al tronco y a las extremidades, respetando la cara, las palmas y las plantas. El diagnóstico final se hizo con base en la clínica y los paraclínicos ordenados, donde se descartó compromiso extracutáneo por medio de resonancia magnética cerebral y de columna total contrastada, fundoscopia y ecografía ocular.

PALABRAS CLAVE: Facomatosis pigmentovascular; Malformaciones vasculares; Síndromes neurocutáneos.

1. Pediatra, Residente de Endocrinología Pediátrica, Departamento de Pediatría, Facultad de Medicina, Universidad de Antioquia y Hospital Universitario San Vicente Fundación. ORCID https://orcid.org/oooo-0oo3-0060-5512

2. Médico, residente de Dermatología, sección de dermatología, facultad de Medicina, Universidad de Antioquia y Hospital Universitario San Vicente Fundación. ORCID https://orcid.org/oooo-ooo1-6593-4275

3. Dermatóloga, docente de Dermatología Pediátrica, sección de dermatología, facultad de Medicina, Centro de Investigaciones Dermatologicas CIDERM, Universidad de Antioquia y Hospital Universitario San Vicente Fundación.

ORCID https://orcid.org/o0oo-0002-9570-2392

Correspondencia: María del Pilar Pereira-Gómez; email: pilipereira17@gmail.com

Recibido: $12 / 11 / 19$; aceptado: $08 / 07 / 20$

Cómo citar: Pereira, MdP; Martínez, M; Mejía, MN. Facomatosis pigmentovascular tipo cesioflamea: a propósito de un caso. Rev Asoc Colomb Dermatol. Vol 28(4): octubre - diciembre, 2020, 352-356.

DOI: https://doi.org/10.29176/2590843X.1554

Financiación: ninguna, conflictos de interés: ninguno 


\section{PHACOMATOSIS PIGMENTOVASCULARIS OF CESIOFLAMMEA TYPE: CASE REPORT}

\section{SUMMARY}

Phacomatosis pigmentovascularis of cesioflammea type is characterized by the presence of a superficial capillary vascular malformation that coexists on the same skin surface with dermal melanocytosis or nevus anemicus. In turn, this entity may be associated with alterations of a systemic nature, especially of the central nervous system. Its incidence and prevalence are unknown due to its low frequency; however, it is of great importance for health professionals involved in newborn care. The case of a newborn of 5 days of life is described below, who was born with a large blue-greyish macule superimposed on an erythematous-violet macule that affected the trunk and extremities, respecting the face, palms and soles. The final diagnosis was made based on the clinic and the ordered paraclinics, where it was ruled out an extracutaneous involvement by means of cerebral magnetic resonance and total contrasted spine, fundoscopy and ocular ultrasound.

KEY WORDS: Phacomatosis pigmentovascularis; Vascular malformations; Neurocutaneous syndromes.

\section{INTRODUCCIÓN}

Las malformaciones vasculares de tipo capilar (MVC) son defectos localizados de la morfogénesis vascular, probablemente causados por una disfunción de las vías que regulan la formación de los canales vasculares durante el desarrollo embrionario, especialmente los nervios vasomotores. En algunos casos, estas malformaciones coexisten sobre la misma superficie cutánea, con melanocitosis dérmicas (MD) u otros hamartomas cutáneos, configurando así las facomatosis pigmentovasculares (FPV). Tradicionalmente se clasifican en cinco tipos: I (MVC + nevo epidérmico), II (MVC + MD o nevo anémico), III (MCV + lentigo [nevus spilus] o nevo anémico), IV (MVC + MD + lentigo o nevo anémico) y V (piel marmórea [cutis marmorata] telangiectásica congénita + MD). En 2005 se propuso otra clasificación agrupándolas en solo 3 tipos: facomatosis pigmentovascular cesioflammea, spilorosea y cesiomarmorata. Además, se realizó una clasificación para diferenciar los que tenían o no compromiso sistémico, siendo acuñados con la letra $(a)$ los pacientes con compromiso cutáneo aislado y con la letra $(b)$ los que tienen algún hallazgo extracutáneo relacionado con trastornos asociados a las facomatosis pigmentovasculares ${ }^{(1,2)}$.

La importancia del diagnóstico de las FPV en los recién nacidos radica en su relación con malformaciones y alteraciones del sistema nervioso central debido a su origen común desde la etapa embrionaria en el ectodermo, específicamente en la cresta neural. Es por esto por lo que dermatólogos, pediatras y neonatólogos deben estar familiarizados con estas entidades, realizando los estudios pertinentes y un diagnóstico oportuno ${ }^{(3)}$.

A continuación, se realiza la descripción de un caso clínico de una paciente hospitalizada en la unidad de cuidados intensivos neonatales en el Hospital Universitario San Vicente Fundación de la ciudad de Medellín, Colombia. Se presentan los datos clínicos (figura 1) y los estudios imagenológicos (figura 2) realizados en la paciente.

Se firmó el consentimiento informado, se reserva la identidad y se conserva el anonimato de la paciente. Se declara no tener conflictos de interés.

\section{PRESENTACIÓN DEL CASO}

Neonata nacida a término, de 5 días de vida, hospitalizada debido a ictericia por isoinmunización Rh. Valorada por manchas en la piel presentes al nacimiento. $\mathrm{Al}$ examen físico presentaba una mácula azul-grisácea de gran extensión, que afectaba tronco y extremidades, respetando cara, palmas y plantas, con superposición de máculas eritematovioláceas, de bordes regulares, 
que desaparecían a la digito presión, sin presencia de thrill, localizadas en el dorso de la espalda y la región lumbar (figura 1).

Con los anteriores datos se consideró que el cuadro clínico de la paciente correspondía a una facomatosis pigmentovascular tipo cesioflamea IIa (mancha mongólica extensa o aberrante más una malformación vascular capilar).

Se solicitó evaluación por neurología infantil, donde descartaron alteraciones neurológicas o melanosis neurocutánea a través del examen físico neurológico y con imágenes por resonancia magnética del sistema nervioso central (cerebral y columna total) (figura 2). La paciente también fue evaluada por oftalmología pediátrica, que descartó compromiso ocular al examen físico oftalmológico y con la realización de una ecografía ocular.

\section{DISCUSIÓN}

Las FPV fueron descritas por primera vez en 1947 por Ota ${ }^{(4)}$; desde entonces, se han reportado aproximadamente 176 casos en la literatura internacional ${ }^{(5)}$. En
Colombia, en un estudio descriptivo sobre dermatosis neonatales en 340 recién nacidos, no se reportó ningún caso sobre estas entidades ${ }^{(6)}$. Solo existe un registro publicado en la literatura nacional de un niño de 3 años, que de forma concomitante tenía un síndrome de Klippel-Trénaunay ${ }^{(7)}$. Las FPV se caracterizan por malformaciones vasculares capilares en coexistencia con melanocitosis dérmicas $\mathrm{u}$ otros hamartomas, con una clasificación que ha cambiado con el tiempo. Las más conocidas son las de 5 y 3 tipos, descritas previamente, en las que se incluye la facomatosis pigmentovascular cesioflamea, variedad diagnosticada en este caso. A pesar de tratarse de alteraciones congénitas, la edad del diagnóstico es variable, con rangos que van desde los 7 días de vida hasta los 78 años, lo cual puede explicarse por el poco conocimiento sobre estas genodermatosis ${ }^{(8)}$. En nuestro caso, el diagnóstico se hizo a los 5 días de vida. En cuanto a las alteraciones genéticas subyacentes, alrededor de 12 pacientes han sido examinados a través de técnicas de secuenciamiento genético, en quienes se han encontrado mutaciones en los genes GNAQ y GNA11, que muestran mosaicismos detectados únicamente en biopsias de piel y no en estudios de sangre periférica. Al parecer, el momento del desarrollo embrionario en que se da la mutación somática es más importante que el tipo de mutación, ya que

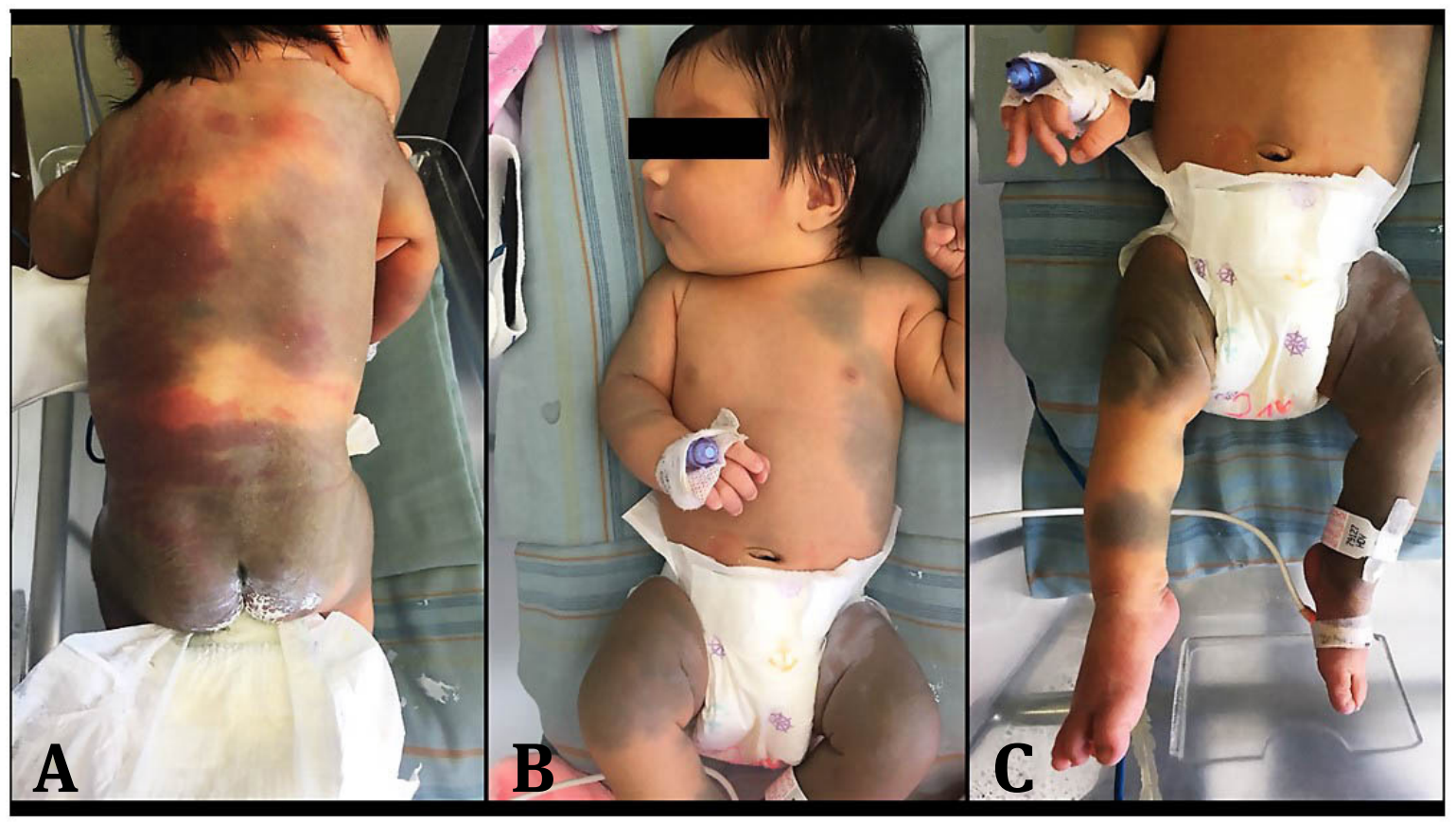

Figura 1. A) Mácula azul de gran extensión, con superposición de mácula eritematosa y violácea, sin thrill que desaparece a la digitopresión. B-C). Mácula azul grisácea de gran extensión en tronco y extremidades. 


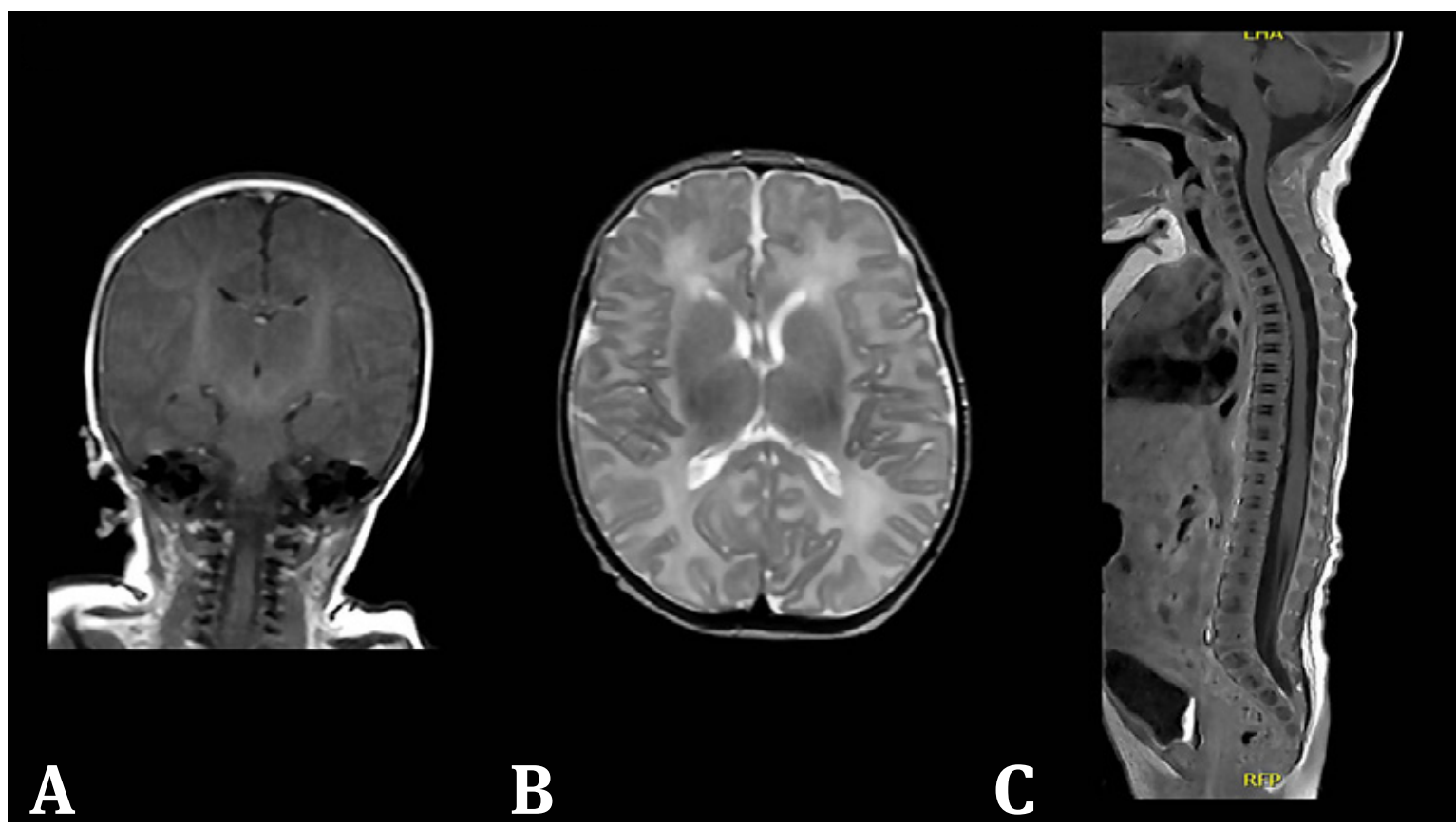

Figura 1. Resonancia magnética cerebral y de columna total contrastada. A) T2, corte transversal de cráneo. B) T1, corte coronal de cráneo. C) T1, corte sagital de columna completa. No se evidencian malformaciones ni melanosis leptomeníngeas.

\section{Puntos clave}

- La facomatosis pigmentovascular tipo cesioflámea se caracteriza por la presencia de una malformación vascular capilar superficial, que coexiste en la misma superficie cutánea con una melanocitosis dérmica o un nevo anémico.

- La importancia del diagnóstico de las FVP en los recién nacidos radica en su relación con malformaciones y alteraciones del sistema nervioso central debido a su origen común desde la etapa embrionaria en el ectodermo.

- Es importante el seguimiento clínico, ya que la mayoría de las lesiones persisten sobre el tiempo, el melanoma uveal y el glaucoma han sido hallazgos encontrados en pacientes durante su seguimiento.

- Es relevante que todo paciente con diagnóstico de FVP sea evaluado por neurología infantil y oftalmología pediátrica y que se le practique una resonancia magnética simple y contrastada del sistema nervioso central con el objetivo de descartar alteraciones extracutáneas.

estas alteraciones genéticas tienen manifestaciones clínicas que se sobreponen entre sí. En la paciente reportada no fue posible realizar estos estudios por falta de disponibilidad de estas pruebas.

Se han descrito asociaciones extracutáneas como: epilepsia, retraso psicomotor, atrofia cerebral, calcifi- caciones cerebrales, ventriculomegalia, hidrocefalia, melanocitosis conjuntival, melanosis ocular, mamilaciones del iris, glaucoma, agenesia renal, alopecia, síndrome de Klippel-Trénaunay, de Sturge-Weber, entre otros ${ }^{(3,5)}$. En esta paciente se descartó compromiso extracutáneo luego de una valoración neurológica y oftalmológica especializada. El compromiso 
extracutáneo es variable en las diferentes series de casos. Entre un 30\% y un 50\% de los casos de FPV cesioflamea y casi un $100 \%$ de las FPV cesiomarmorata tienen alguna alteración en otros órganos; sin embargo, esto puede estar sobreestimado por el tipo de publicación y los sesgos de lugar, ya que la mayoría de los casos reportados fueron diagnosticados en centros especializados $^{(9)}$.

El seguimiento clínico es importante, ya que la mayoría de las lesiones persisten sobre el tiempo. Se han reportado 8 casos de melanoma uveal en pacientes entre los 30 y 80 años, donde todos tenían el antecedente común de una FPV con melanocitosis ocular ${ }^{(10)}$. Igualmente se ha descrito mayor incidencia de glaucoma en adolescentes y adultos jóvenes con afectación importante de la visión, que ha requerido manejo especializado ${ }^{(11,12)}$.

En relación con el tratamiento, no hay terapia curativa; generalmente es de índole estético, con resultados variables, con el uso de láser rubí Q-switched para las lesiones pigmentadas y de láser de colorante pulsado para las lesiones vasculares ${ }^{(3)}$.

\section{CONCLUSIÓN}

Presentamos el caso de una recién nacida con una facomatosis pigmentovascular (FPV) cesioflamea tipo IIa, una entidad poco frecuente, pero con gran relevancia clínica en el seguimiento y pronóstico de los pacientes. A raíz del caso y de la revisión realizada, sugerimos que todo paciente con diagnóstico de FPV sea evaluado por neurología infantil y oftalmología pediátrica y que se le practique una resonancia magnética simple y contrastada del sistema nervioso central con el objetivo de descartar alteraciones extracutáneas y establecer un seguimiento clínico periódico.

\section{REFERENCIAS}

1. Ünlü E, Şahin T. A Neonatal Case of Phacomatosis Pigmentovascularis Type IIa. Balkan Med J. 2015;32(1):129-30. doi: 10.5152/balkanmedj.2015.15448

2. Püttgen KB, Cohen BA. Neonatal Dermatology. En: Cohen BA (editor). Pediatric Dermatology. 4.a edición. China: Saunders; 2013. p. 14-67. doi: 10.1016/B978-0-7234-3655-3.00002-3

3. Dutta A, Ghosh SK, Bandyopadhyay D, Bhanja D, Biswas S. Phakomatosis Pigmentovascularis: A Clinical Profile of 11 Indian Patients.
Indian J Dermatol. 2019;64(3):217-23. doi: 10.4103/ijd.IJD_385_18

4. Ota M, Kawamura T, Ito N. Phacomatosis pigmentovascularis. Jpn J Dermatol B. 1947;52:1-3.

5. Kumar A, Zastrow DB, Kravets EJ, Beleford D, Ruzhnikov M, Grove M, et al. Extracutaneous manifestations in phacomatosis cesioflammea and cesiomarmorata: Case series and literature review. Am J Med Genet A. 2019;179(6):966-77. doi: 10.1002/ajmg.a.61134

6. Otero-Marrugo V, Bitar-Zapata R, CastellanosLorduy H, Gómez-Agámez A. Dermatosis neonatales: valoración clínica en 340 recién nacidos cordobeses. Rev Asoc Colomb Dermatol Cir Dermatol. 1993;2(4):154-7. https://bit.ly/3903KA3

7. Zapata F. Facomatosis pigmentovascularis asociada a sindrome de Klippel-Trenaunay y a alopecia triangular. Reporte de caso. CES Med. 2012;26(2):229-36. doi: 10.21615/ces\%2omed.v26i2.2318

8. Nanda A, Al-Abdulrazzaq HK, Habeeb YKR, Zakkiriah M, Alghadhfan F, Al-Noum R, et al. Phacomatosis pigmentovascularis: Report of four new cases. Indian J Dermatol Venereol Leprol. 2016;82(3):298-303. doi: 10.4103/0378-6323.178905

9. Fernández M, Boixeda P, de Las Heras E, Aboin S, García C, Olasolo P. Phakomatosis pigmentovascularis: Clinical findings in 15 patients and review of the literature. J Am Acad Dermatol. 2008;58(1):8893. doi: 10.1016/j.jaad.2007.08.012

10. Shields CL, Nicola MDI, Pellegrini M, Shields JA. Choroidal melanoma in phakomatosis pigmentovascularis with Klippel-Trenaunay syndrome. Retina. 2018;38(11):2220-7. doi: 10.1097/IAE.oooooooooooo1856

11. Van der Merwe E, Bhika R, Meyer D. Glaucoma in Phacomatosis Pigmentovascularis in a Young African Adolescent Boy: A Case Report. J Glaucoma. 2019;28(7):e124-5. doi: 10.1097/IJG.0000000000001264

12. Singh K, Dangda S, Mutreja A, Bhattacharyya M, Jaisingh K. Bilateral Phacomatosis Pigmentovascularis in a Young Male with Developmental Glaucoma and Varicose Veins. J Curr Glaucoma Pract. 2018;12(2):94-98. doi: 10.5005/jp-journals-10008-1251 